\title{
PLANIFICAÇÃO DE PEÇAS OBTIDAS PELA INTERSEÇÃO DE SUPERFÍCIES CILINDRICAS
}

\author{
PLANNING FOR PIECES OBTAINED BY THE INTERSECTION OF CYLINDRICAL SURFACES \\ Marcelo Lacortt, Neuza Terezinha Oro \\ Professores do Instituto de Ciências Exatas e Geociências, Universidade de Passo Fundo, Campus I, Bairro São José, BR- 285, \\ CEP: 99052-900 - Cx. Postal 611, E-mail: lacortt@upf.br; neuza@upf.br
}

\begin{abstract}
RESUMO
Este artigo apresenta a modelagem matemática desenvolvida para a planificação de peças cilíndricas, sendo estas peças formadas pela interseção de dois cilindros, com o objetivo de elaborar algoritmos, que implementados em linguagem Pascal, originam dois programas com a finalidade de automatizar a sua planificação. O primeiro programa automatiza a planificação de peças formadas por cilindros perpendiculares entre si, já o segundo programa automatiza a planificação de peças formadas por cilindros oblíquos entre si, sendo este com qualquer ângulo de inclinação entre os cilindros, inclusive $90^{\circ}$ entre si, que é o caso do primeiro programa. Os resultados obtidos pelo programa também estão expostos no decorrer do artigo, sendo que no final tem uma representação plana de cada caso.
\end{abstract}

Palavras chaves: Modelagem Matemática. Automatização. Planificação.

\begin{abstract}
This paper presents mathematical modeling developed for the planning of cylindrical pieces, these pieces being formed by the intersection of two cylinders, with the goal of developing algorithms that implemented in Pascal, originate two programs in order to automate their planning. The first program automates the planning of pieces formed by rollers mutually perpendicular, since the second program automates the planning of pieces formed by cylinders oblique to each other, this being at any angle of inclination between the cylinders, even $90^{\circ}$ between themselves, which is if the first program. The results obtained by the program are also exposed throughout the article, and the end has a planar representation of each case.
\end{abstract}

Keywords: Mathematical Modeling. Automation. Planning.

\section{INTRODUÇÃO}

O desenvolvimento da superfície de uma peça a ser construída é de suma importância para um dos ramos da indústria metalúrgica denominada de caldeiraria industrial, já que as peças utilizadas são geralmente de grande porte e de várias formas e dimensões. Dessa forma, é importante que a tarefa de resolvê-las seja realizada de modo rápido e preciso.

Com a globalização, as exportações estão em alta e o mercado está repleto de fornecedores disputando espaços de forma acirrada, onde a inovação é o diferencial responsável pelo sucesso e permanência destas indústrias no mercado. Neste contexto, a tecnologia é uma grande aliada nesse processo e está, nesse momento, em grande ascendência principalmente no ramo da metalurgia, norteando a produção de peças, onde, com auxílio de softwares, desenha, recorta e monta automaticamente as peças a serem comercializadas pela indústria. 
Para construir um software é necessário modelar as peças e elaborar algoritmos a partir destes modelos para implementação, de forma automatizar o processo de fabricação de peças. Algumas dessas peças podem ser entendidas como interseção entre sólidos geométricos.

O objetivo geral deste trabalho é desenvolver algoritmos para implementação computacional, através de modelos matemáticos que generalize a obtenção das coordenadas espaciais dos sólidos, obtidos pela interseção entre dois cilindros, e das coordenadas que as planifiquem.

\section{REFERENCIAL TEÓRICO}

A caldeiraria industrial utiliza-se de chapas planas para a fabricação de vários tipos de peças como cones, cilindros, transições, etc., que unidas resultam em tubulações e outros tipos de construção em chapa. A obtenção destas peças é a partir das operações de dobramento e curvamento das chapas até a obtenção das peças desejadas. Porém, para obter-se uma peça, deve-se determinar a forma que a chapa plana deve ser cortada, para que após as operações de fabricação, a peça possa ser obtida. A obtenção da forma plana da superfície lateral é denominada de planificação da superfície.

Tradicionalmente a determinação da planificação da superfície era feita com a confecção de modelos em papelão, que após eram recortados e transferido o contorno para a chapa. Para isso, era necessário um projetista com conhecimentos da técnica para a obtenção do modelo em papelão.

O uso de software permite a obtenção da planificação da superfície de uma peça com maior facilidade e precisão, bem como o conhecimento necessário para o uso do software se restrinja ao aprendizado do mesmo e o conhecimento da área onde a peça será utilizada, além de conhecimento dos processos de fabricação.

Neste sentido, a modelagem matemática, como processo para a obtenção de modelos que descrevam a planificação de superfícies de peças, faz parte da concretização de um trabalho interdisciplinar. Entende-se por modelagem matemática como um processo matemático elaborado a partir do estudo de um problema real, o qual tem como finalidade descrever a realidade através de um modelo matemático abstrato e, assim, obter suporte para sua representação simplificada, solução e análise. (GOLDBARG \& LUNA, 2005).

Além de possibilitar investigar recursos matemáticos adequados para a solução de um problema específico, a modelagem matemática possibilita também a construção de estruturas matemáticas que podem ser aplicadas em outras situações problema de natureza semelhante. Segundo Biembengut, "a modelagem matemática é, assim, uma arte, ao formular, resolver e elaborar expressões matemáticas que valham não apenas para uma solução particular, mas que também sirvam, posteriormente, como suporte para outras aplicações e teorias" (1999).

Ainda de acordo com Biembengut, para o desenvolvimento do processo de modelagem matemática são necessários alguns procedimentos, que podem ser agrupados em três etapas: a interação, matematização e modelo matemático.

A interação é entendida como reconhecimento da situação-problema, ou seja, a familiarização com o assunto a ser modelado. Já a matematização é a parte mais complexa do processo de construção de modelo, uma vez que será formulado e resolvido o problema. Nesta etapa se traduz a situação-problema para uma linguagem matemática, mas para isso o indivíduo tem de ter um aguçado conhecimento sobre as entidades matemáticas usadas na formulação. A informática também é imprescindível nesta etapa, com seus softwares, para a resolução automática dos problemas matemáticos obtidos. E o modelo matemático é a etapa em que se dá interação da solução, ou seja, uma investigação para verificar o nível da aproximação da situação-problema em relação à realidade, avaliando quão significativa e relevante é a 
solução e qual sua veracidade, em outras palavras, se a aproximação do resultado condiz com a realidade. (BIEMBENGUT, 1999).

Ainda, Bassanezzi (2002) afirma que o modelo matemático é a representação de um sistema, ou seja, um conjunto de símbolos e relações matemáticas que representam de alguma forma o objeto estudado. Dessa forma, um modelo matemático é uma representação abstrata, ou uma aproximação, de um problema real. Tal representação pode ser expressa em termos de expressões matemáticas (equações), por meio de uma série de células inter-relacionadas em uma planilha de cálculo, entre outras. Em qualquer que seja o caso, o propósito de um modelo matemático é representar a essência de um problema de forma concisa. Isso traz uma série de vantagens, como permitir ao analista uma melhor compreensão do problema em estudo.

Nesta perspectiva, o desenvolvimento de uma modelagem matemática para a planificação de peças cilíndricas intersecionadas é fundamental, uma vez que a automação do processo de obtenção dessas peças torna-se possível, fazendo com que a planificação das superfícies dos sólidos possa se converter num processo preciso, fácil e rápido de ser executado. (BESANT, 1988)

Os problemas de planificação de superfícies não são de hoje, sendo que as soluções foram desenvolvidas em função das tecnologias disponíveis em cada época. Vários estudiosos desenvolveram metodologias para obtenção de planificação de peças, entre eles podemos citar Cookson (1964), que apresenta em sua obra fórmulas matemáticas para obtenção de verdadeiras grandezas para casos específicos de peças, além dos métodos manuais de traçado, baseados na Geometria Descritiva.

Já Araújo (1976) apresenta resolução gráfica para o desenvolvimento de peças mais usualmente trabalhadas no campo da funilaria, da caldeiraria e da manutenção industrial. No entanto, no caso de peças mais elaboradas, não apresenta uma metodologia.

As dificuldades e erros ocorridos nas execuções de trabalhos caros e complexos estimularam Castro (1991) a resolver, os problemas de planificação de forma analítica e computacional. O autor descreve um método analítico para planificação de interseções entre superfícies cilíndricas e cônicas, apresentando a determinação das equações matemáticas das linhas de corte, que devem ser traçadas nas superfícies planificadas.

Um algoritmo de planificação genérico foi desenvolvido por Oro et al (2000), na Universidade de Passo Fundo. Os autores apresentam o desenvolvimento de um algoritmo, obtido através da modelagem matemática do problema de planificação de superfícies de corpos cilíndricos e cônicos, utilizando-se para isso do método de triangulação. A partir do sólido modelado em 3D, é mapeada a superfície pelo método de triangulação ${ }^{1}$ para obter a planificação da superfície do sólido. A planificação obtida também utiliza o método de triangulação para calcular a posição dos pontos no plano. $\mathrm{O}$ algoritmo elaborado executa a planificação de forma genérica. Inicialmente, o algoritmo calcula as coordenadas espaciais que definem a superfície lateral da peça a ser planificada, de forma ordenada e, em seguida, o processo de planificação realizado é o mesmo para qualquer superfície lateral considerada. Observa-se que a principal diferença entre o método proposto e os demais existentes está efetivamente no processo de planificação. O método proposto consiste na planificação de pontos, realizando a transformação de pontos do espaço para o plano. Os demais métodos anteriormente citados trabalham com procedimentos de planificação específicos para peças específicas. O algoritmo foi implementado, resultando num software que foi denominado por "Planificação de Superfícies".

Pertence (2000) apresenta a implementação de um software didático em linguagem Visual Basic que permite obter a planificação de sólidos. Ele utiliza expressões matemáticas que resultam da análise das

\footnotetext{
${ }^{1}$ O Método de Triangulação: da Descritiva tradicional consiste em discretizar a superfície lateral do sólido através de " $2 n$ " triângulos e posteriormente realizar a planificação dos pontos do espaço que definem os vértices dos triângulos.
} 
projeções de primitivas básicas tais como cilindros e cones, obtendo equações correspondentes às coordenadas das bases superior e inferior que formam a superfície lateral do sólido.

Além disso, Maziero et al (2003), apresenta a implementação do software mencionado anteriormente em um sistema CAD comercial (AutoCAD). Este aplicativo permite a modelagem de peças em 3D, peças estas disponibilizadas na interface gráfica. Após a modelagem em 3D, o usuário pode solicitar a planificação da referida peça. Este sistema permite a modelagem de diversas peças, as quais são criadas em camadas (layers) diferentes, permitindo a sua individualização. Apresenta, também, a metodologia de desenvolvimento da interseção de sólidos por planos oblíquos. Este método permite truncar o sólido e identificar a peça resultante que se deseja obter, representar a peça em um modelo 3D, e obter a planificação da superfície da peça resultante. Permite ainda, que a peça resultante possa ser secionada outras vezes e também possam ser obtidas as planificações das superfícies destas peças resultantes.

O software comercial CALDSOFT (2013), permite executar a planificação da superfície de peças predefinidas no sistema. O mesmo não permite a obtenção da planificação de uma peça criada a partir de novas definições, ou seja, a partir de uma peça modelada em 3D obter a sua planificação.

\section{MODElageM MATEMÁtiCa do PROCESSO DE PLANIFICAÇÃO DE PEÇAS OBTIDOS A PARTIR DA INTERSEÇÃO ENTRE DOIS CILINDROS}

A modelagem matemática a ser descrita neste trabalho foi fundamentada nos trabalhos citados no item anterior, tendo como principal base os trabalhos de "Planificação de Superfícies" desenvolvido por Oro et al (2000) e por Maziero et al (2003), que tem por finalidade desenvolver um algoritmo genérico, que implementado em uma linguagem de programação, planifica diversos casos de peças com as características geométricas estudadas.

O diferencial deste trabalho em relação ao citado anteriormente está na construção do modelo, que foi embasada na correspondência entre princípios da geometria descritiva, trigonometria, geometria analítica plana e espacial, além de apresentar a planificação das duas peças obtidas na intersecção dos dois cilindros. O modelo espacial, e posterior planificação, desenvolvido Oro et al (2000) e, especialmente, por Maziero et al (2003) é construído somente para uma das peças obtidas na intersecção, a peça representada por um cilindro horizontal e a peça representada pelo cilindro oblíquo.

A elaboração do modelo foi dividida em duas partes: a primeira corresponde à representação da superfície lateral do sólido no espaço e a segunda, ao cálculo dos pontos no plano correspondentes à superfície lateral planificada.

\subsection{Planificação de cilindros perpendiculares entre si}

Considando dois cilindros perpendiculares entre si (ver Figura 1), onde através da geometria e trigonometria foram calculadas suas coordenadas no espaço e após as coordenadas no plano, conforme segue. 


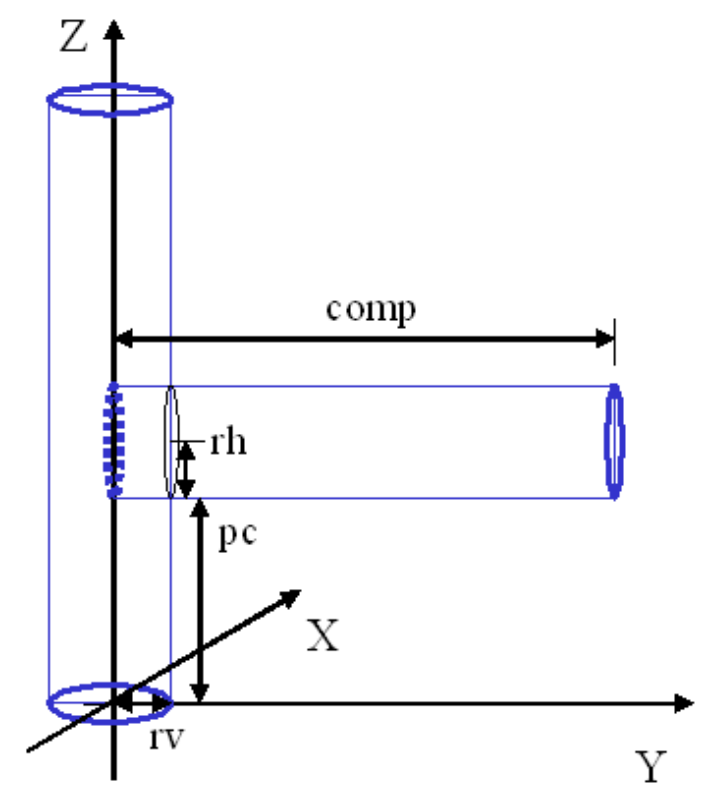

Figura 1. Cilindros perpendiculares entre si.

O número de divisões para o cilindro vertical determina a precisão de sua superfície, o qual é composta por geratrizes, visto que o número de pontos para a base superior e para a intersecção coincidem. Nesse trabalho, o número de pontos tomados sobre a circunferência que formam as bases do cilindro foram doze, conforme Figura 2, pois com este número de pontos sua representação no espaço e no plano é mais precisa e, também, seria fácil verificar no desenho se as coordenadas calculadas pelo modelo estavam corretas.

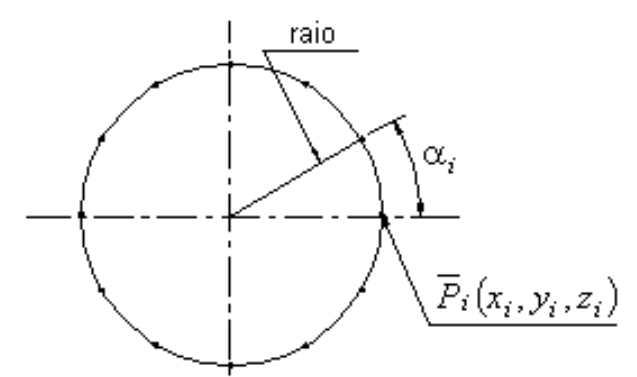

Figura 2. Divisões de uma das bases do cilindro.

Quanto ao sistema de coordenadas, a origem fica no cruzamento das linhas de centro dos dois cilindros, ou seja, o eixo $y$ fica na linha de centro do cilindro horizontal o eixo $z$ está na linha de centro do cilindro vertical, quando este está centrado com do cilindro horizontal, e o eixo $x$ marca o deslocamento $\Delta x$ cilindro vertical, que nesse trabalho $\Delta x=0$. A figura 1 mostra a origem do sistema de coordenadas e como os eixos se orientam.

Para obter estas coordenadas em um espaço tridimensional $(x, y, z)$, inicialmente os cilindros foram considerados separados, um vertical e outro horizontal e após foram determinadas as coordenadas 
de interseção entre ambos. Dessa forma, as coordenadas dos cilindros foram divididas em dois planos bidimensionais $(x, y) \mathrm{e}(y, z)$.

As coordenadas espaciais do cilindro vertical $P V\left(x_{v}, y_{v}, z_{v}\right)$, cujas bases são circunferências, foram calculadas através da trigonometria em um plano $(x, y)$, sendo:

$$
\begin{aligned}
x_{v i} & =\text { raio. } \cos \alpha_{i} \\
y_{v i} & =\text { raio.sen } \alpha_{i} \\
\alpha_{i} & =(i-1) \cdot\left(\frac{2 \pi \cdot \text { raio }}{12}\right)
\end{aligned}
$$

sendo $i=1,2, . ., 12$, a base superior assumida como altura $z_{v}$ e a inferior a altura é nula (ver Figura 1).

As coordenadas espaciais do cilindro horizontal $P E\left(x_{E}, y_{E}, z_{E}\right)$ e $P D\left(x_{D}, y_{D}, z_{D}\right)$, mostrado na Figura 1, cujas bases também são formadas por duas circunferências, foram calculadas de forma semelhante a do cilindro vertical só que em um plano $(x, z)$, e com $z$ adicionado a uma altura considerada ponto de interseção entre os dois cilindros mais o raio do cilindro vertical, e após a base direita deslocada a um comprimento $y$.

Com as coordenadas espaciais dos dois cilindros calculadas passou-se ao cálculo dos pontos de intersecção entre os dois cilindros. Primeiramente foi tentada a obtenção destas coordenadas através da intersecção entre as geratrizes do cilindro vertical com o horizontal, a qual não teve sucesso, por serem ortogonais.

Após várias tentativas, foram obtidas as coordenadas espaciais dos pontos de interseção, através do equacionamento da circunferência do cilindro vertical, em função do $x$ do cilindro horizontal, conforme as relações (4), (5) e (6).

$$
\begin{aligned}
y_{I i} & =\sqrt{\text { raio }^{2}-x_{i}^{2}} \\
z_{I i} & =z \text { do cilindro horizontal } \\
x_{I i} & =x \text { do cilindro horizontal. }
\end{aligned}
$$

Obtidos os pontos de intersecção $\left(P_{I i}\right)$ no espaço, calculou-se a distância entre os pontos de intersecção e os pontos da base direita $\left(P_{D i}\right)$ do cilindro vertical, que representam o comprimento das geratrizes $\left(G_{i}\right)$, pela equação (7).

$$
G_{i}=d\left(P_{I i}, P_{D i}\right)
$$

As cordas correspondentes às bases esquerda ( $c e$ ), obtida através do processo de interseção, e direita $(c d)$ são calculadas no espaço através do cálculo da distância entre pontos subsequentes da mesma base, ou seja:

$$
\begin{aligned}
& c e=d\left(\overline{P_{I i}}, \overline{P_{I i+1}}\right) \\
& c d=d\left(\overline{P_{D i}}, \bar{P}_{D i+1}\right)
\end{aligned}
$$


Tomando-se os pontos no espaço como referenciais e utilizando as $G_{i}$, ce e $c d$, passando para próxima etapa que é a planificação do cilindro horizontal. Calcula-se os $n+1$ pontos do plano $P_{i}\left(x_{i}, y_{i}\right)$ correspondentes a eles na planificação da superfície do sólido, observando o fato que devem ser mantidas estas distâncias entre os pontos correspondentes da superfície planificada, conforme Figura 3.

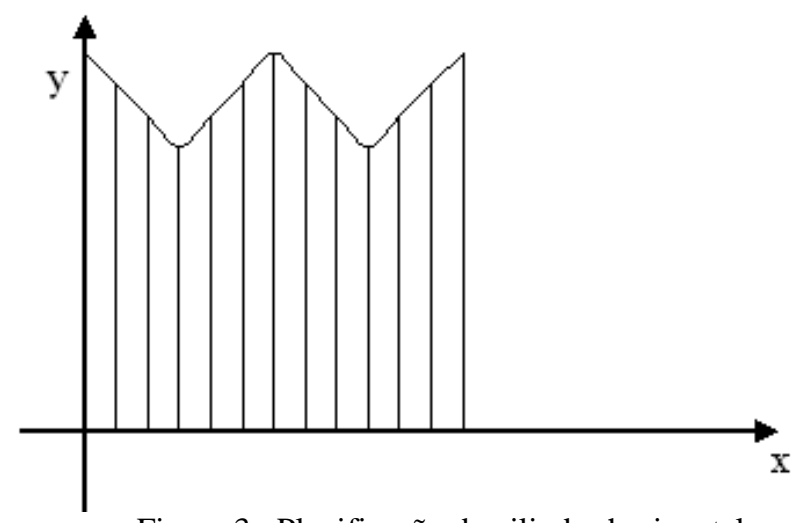

Figura 3: Planificação do cilindro horizontal.

Por convenção, o primeiro ponto da superfície planificada $P_{1}$, correspondente ao primeiro ponto da base direita $\left(P_{D}\right)$, foi localizado na origem do sistema $x y$, ou seja: $P_{1}=(0,0)$; o segundo ponto da superfície planificada $P_{2}$, correspondente ao primeiro ponto da base esquerda $(P I)$ foi localizado no eixo $y$, onde a ordenada do ponto corresponde à primeira geratriz, ou seja, $P_{2}=\left(0, G_{1}\right)$.

Conhecidos os dois primeiros pontos planificados, passou-se ao cálculo dos demais, levando em consideração o fato que esses valores devem se corresponder no espaço e no plano. Sendo conhecidos os pontos $P_{1}$ e $P_{2}$, para determinação dos próximos pontos utilizou-se o procedimento descrito a seguir.

$$
\begin{aligned}
& x_{i}=c d+x_{i-1} \\
& y_{i}=G_{i} \\
& i=2 ., 3, \ldots, 13
\end{aligned}
$$

Já a planificação da peça vertical, mostrada na Figura 4, foi mais trabalhosa por se tratar de obter as coordenadas do furo feito pela intersecção das peças.

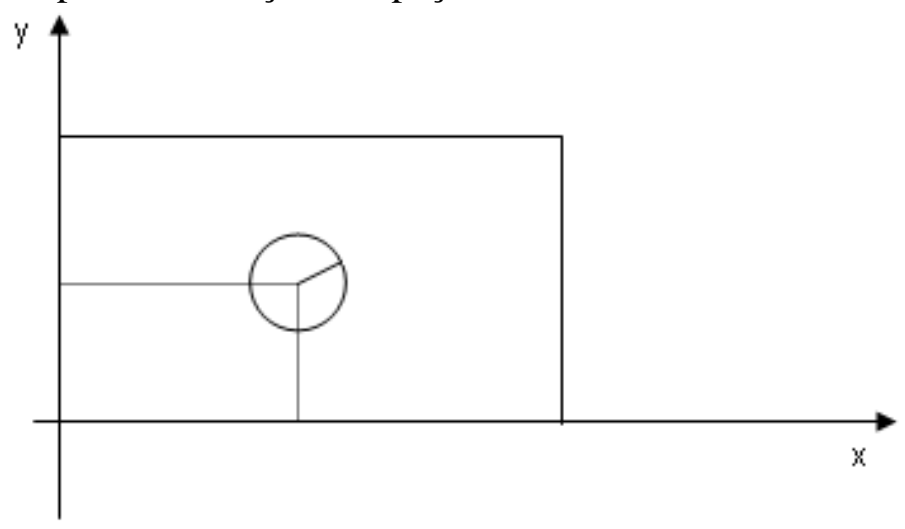

Figura 4: Planificação do cilindro vertical 
Após analisar as dimensões da peça chegou-se à conclusão de que a posição do furo em $x$ poderia ser centralizada e com o comprimento total sendo o perímetro da circunferência partiria com o centro do furo com a medida de meio perímetro de circunferência, ou seja, $\pi \cdot$ raio, tendo o centro para encontrar os valores em $x$ soma-se a $\cos \alpha$. E as dimensões do furo em $y$ têm os mesmos valores de $z_{I}$ (ponto de intersecção entre os cilindros).

Os extremos do cilindro vertical foram fáceis de serem obtidos, pois forma um retângulo, onde quatro pontos o descrevem no plano, sendo os pontos inferiores $(0,0)$ e $(2 \pi . r, 0)$ e superiores $(0$,altura) e $(2 \pi . r$, altura $)$.

\subsection{Planificação de Cilindros oblíquos entre si.}

A próxima peça a ser planificada foi àquela obtida pela interseção entre um cilindro reto e um oblíquo. A Figura 5 mostra o esquema geométrico usando no processo de modelagem.

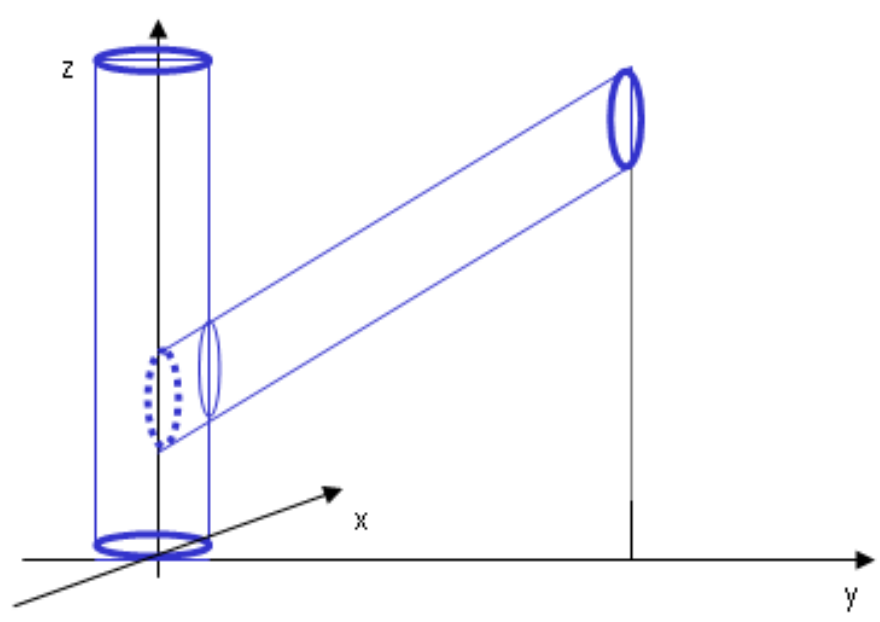

Figura 5. Cilindros oblíquos entre si.

A modelagem do anterior já não serve para este caso, visto que neste caso para determinar suas coordenadas no espaço temos de levar em conta o ângulo de inclinação entre os cilindros.

O trabalho inicia com a retirada de dados de um modelo de desenho plano (Ver Figura 6). 


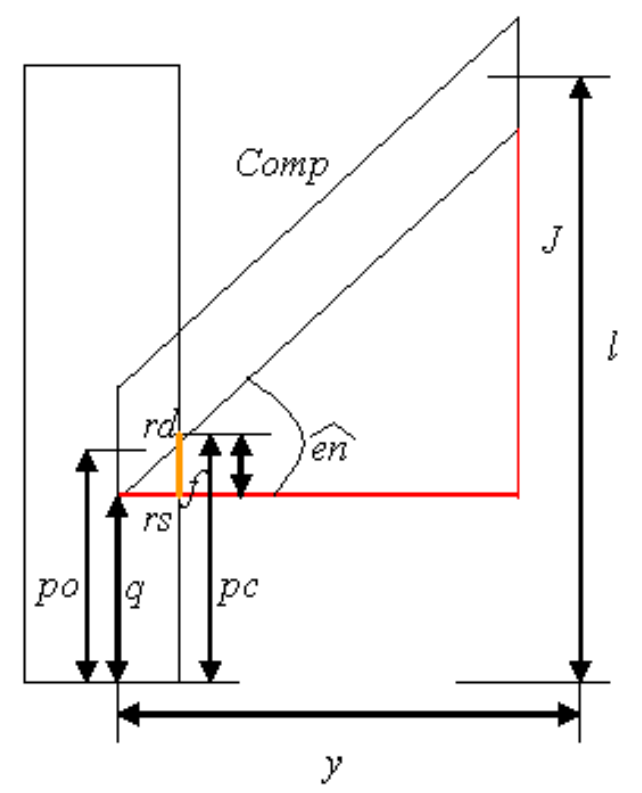

Figura 6: Esquema plano dos cilindros oblíquos entre si.

Sendo que: ên representa o ângulo de inclinação a ser fornecido; $p c$ a altura do ponto de corte a ser fornecido e Comp o comprimento a se fornecido. As demais medidas são fornecidas pelas seguintes expressões.

$y=$ Comp.cos(en)

$f=r s . \operatorname{tg}(e n)$

$q=p c-f$

$p o=q+r d$

$j=\operatorname{Comp} \cdot \operatorname{sen}($ en $)$

$l=j+p o$

Com estes dados, foram obtidas as coordenadas dos cilindros no espaço, sendo as do cilindro vertical foi usado o mesmo processo do item anterior. Já nas coordenadas do cilindro oblíquo, foram utilizadas o mesmo processo do cilindro horizontal anterior para os valores $x$ e $y$, porém para obter os valores $z$, utilizou-se os dados do modelo mostrado na Figura 7, ou seja os valores da coordenada $z$ esquerdo são a distância $q$ e os valores dos $z$ direito são a distância $l$.

Os pontos de intersecção foram semelhantes aos da peça anterior, porém somado aos $z$ a distância $f$. Porém, as coordenados no plano da peça oblíqua foram bem mais complicadas do que a peça horizontal anterior, pois sua base não é reta como a anterior. Para a realização desta planificação foi montado outro modelo, conforme esquema representado na Figura 7. 


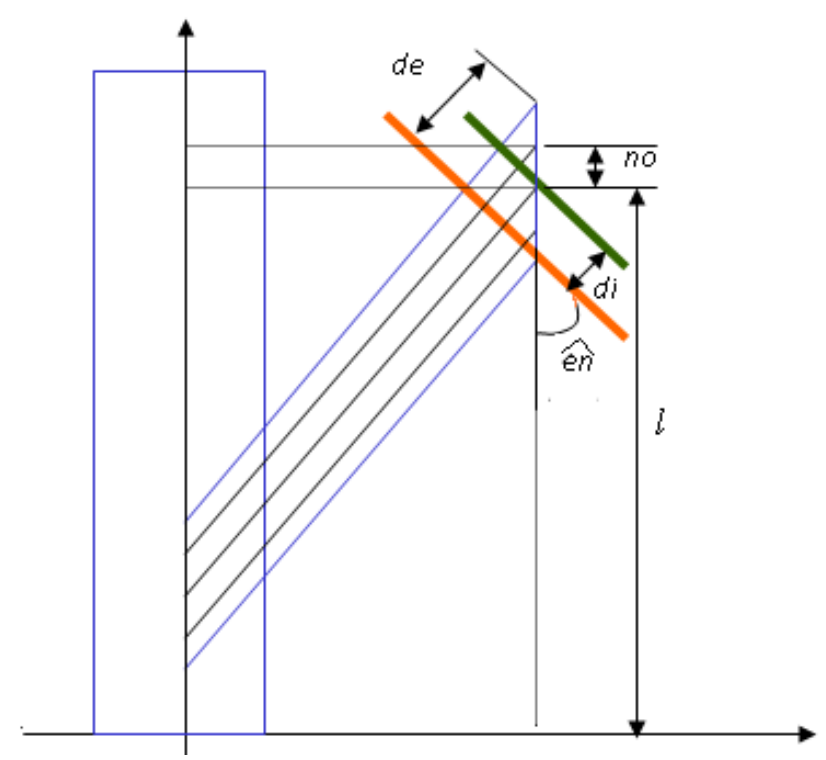

Figura 7: Esquema do modelo de para a obtenção das coordenadas planas.

As medidas $d i$, no e de são obtidas pelas relações:

$d i=\operatorname{sen}($ en $) \cdot n o$

$n o=z d-l$

$d e=n o \cdot \operatorname{sen}(e n)+d i$

Para utilizar as geratrizes na obtenção dos valores de $y$ e as cordas na obtenção dos valores $x$, o seguinte processo foi executado.

Os valores de $x$ as medidas das cordas do cilindro e os valores dos $y$ superiores as geratrizes menos a distância de mais um deslocamento Comp. Já os $y$ inferiores o deslocamento Comp menos a distância $d e$, onde é necessário o deslocamento para utilizarmos os quadrantes em $y$ positivos, pois o programa programado em linguagem Pascal fornece os desenhos somente nos quadrante superiores, conforme esquema representado na Figura 8.

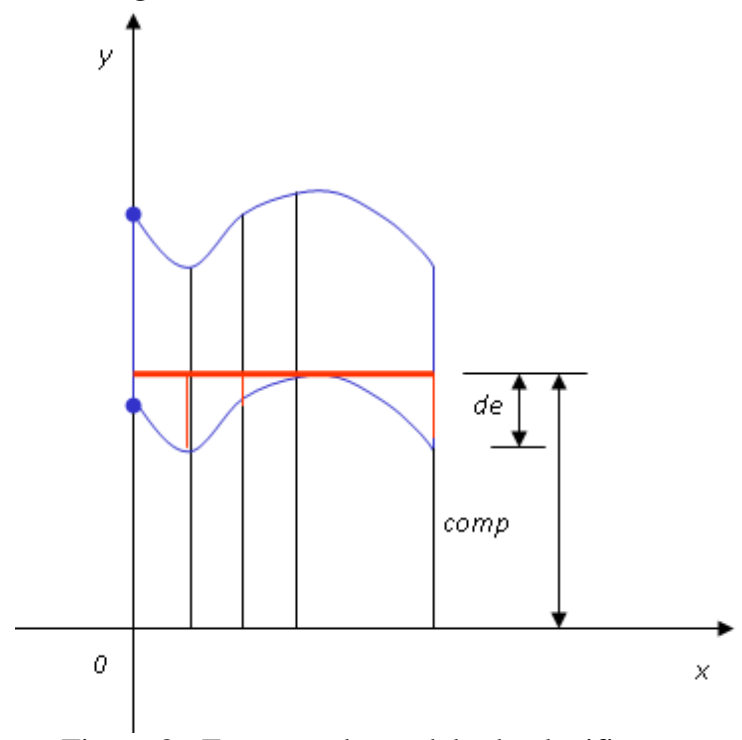

Figura 8: Esquema do modelo de planificação 
Tomando-se os pontos no espaço como referenciais e utilizando as medidas anteriormente definidas, passou-se para próxima etapa que é a planificação do cilindro oblíquo. Calcula-se os $n+1$ pontos do plano $P_{p}\left(x_{i}, y_{i}\right)$ correspondentes a eles na planificação da superfície do sólido, observando o fato que devem ser mantidas estas distâncias entre os pontos correspondentes da superfície planificada.

Assim, para os pontos da base obtida através do processo de interseção $P_{p s}\left(x_{p s}, y_{p s}\right)$ tem-se:

$x_{p s}[1]=0$

$x_{p s}[i+1]=c d[i]+x_{p s}[i]$

$y_{p s}[i]=G[i]-d e[i]+\operatorname{Comp}$

$i=1,2, . ., 12$.

E, para os pontos da outra base, que está à direita (ver Figura 8), $P_{p i}\left(x_{p i}, y_{p i}\right)$ tem-se:

$x_{p i}[1]=0$

$x_{p i}[i+1]=c d[i]+x_{p i}[i]$

$y_{p s i}[i]=\operatorname{Comp}-d e[i]$

$i=1,2, . ., 12$.

\section{RESULTADOS}

Após ter determinado as coordenadas planas dos modelos anteriormente citados, passou-se para a programação em linguagem Pascal 7.0. Os objetivos da realização dessa programação são os de validar e de simular os modelos elaborados, possibilitaram a transposição da superfície da peça do espaço para o plano, isto é a planificação das peças apresentadas neste trabalho. Ou seja, conhecendo os pontos espaciais que descrevem as peças, através do método de triangulação, obter a planificação, com base no modelo desenvolvido por Oro et al (2000).

Na figura 9, representam-se a planificação dos cilindros perpendicilares, onde foram tomadas como medidas do cilindro vertical um raio de $20 \mathrm{~mm}$ e um comprimento de $150 \mathrm{~mm}$, já do cilindro vertical foram tomadas como medidas um raio de $30 \mathrm{~mm}$ e uma altura de $200 \mathrm{~mm}$ e ponto de corte entre os cilindros 50mm. Especificamente, na Figura 9 (a) está mostrada a planificação do cilindro horizontal e na Figura 9 (b) a planificação do cilindro vertical. 


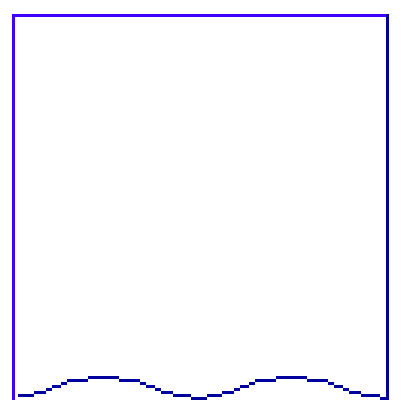

(a) Cilindro horizontal

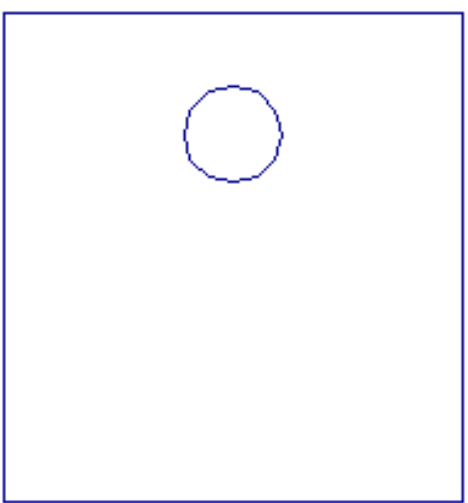

(b) Cilindro vertical

Figura 9: Planificação de cilindros perpendiculares

Representam-se, na Figura 10, a planificação dos cilindros oblíquos, nos quais, como os cilindros anteriores, foram tomadas como medidas do cilindro obliquo um raio de $20 \mathrm{~mm}$ e um comprimento $150 \mathrm{~mm}$, já do cilindro vertical foram tomadas como medidas um raio de $30 \mathrm{~mm}$ e uma altura de $200 \mathrm{~mm}$. Como ponto de corte entre os cilindro foram tomadas uma distância de $50 \mathrm{~mm}$ e como inclinação entre os cilíndros um ângulo de $45^{\circ}$. Na Figura 10 (a) está mostrada a planificação do cilindro oblíquo e na Figura 10 (b) a planificação do cilindro vertical

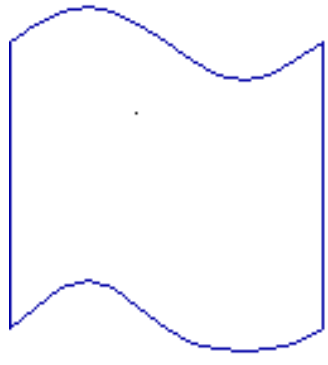

(a) Cilindro oblíquo

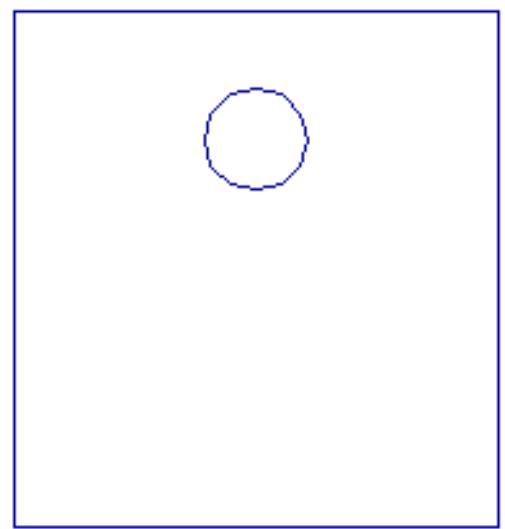

(b) Cilindro vertical

Figura 10. Planificação de cilindros oblíquos.

Inicialmente foram feitos os cálculos manualmente para um número reduzido de pontos, após foi feito o cálculo das mesmas coordenadas através do programa. Por isso, pode-se afirmar que a modelagem matemática das peças específicas mostrou-se eficiente com relação a planificação de peças cilíndricas e estão de acordo com os resultados esperados, porém foi trabalhosa a dedução das situações de interseção. 


\section{CONSIDERAÇÕES FINAIS}

A modelagem matemática é fundamental na elaboração de algoritmos para programação e as indústrias de caldeiraria são cada vez mais beneficiadas com este ramo de pesquisa, com a tecnologia levando a estas indústrias mais agilidade e qualidade em suas produções de peças.

$\mathrm{O}$ trabalho aqui desenvolvido visando à planificação de peças cilíndricas para, posteriormente, serem montadas mostrou-se eficaz em seus objetivos. Com isso, foi elaborado dois programas computacionais que, implementados em linguagem Pascal, planificam peças formadas pela intersecção de dois cilindros, bastando oferecer dados como comprimento, altura, raio e inclinação entre os cilindros que formam a peça.

Apesar de aparentar uma determinada complexidade na modelagem matemática desenvolvida para a obtenção destes algoritmos, foram utilizados conceitos básicos a nível superior, como trigonometria e geometria espacial e analítica.

O primeiro estudo de caso gerou um programa que planificava apenas peças cilíndricas formadas por cilindros perpendiculares entre si, já o segundo planifica peças formadas por cilindros com qualquer ângulo de inclinação entre si, sendo estes oblíquos ou perpendiculares entre si, satisfazendo as necessidades do primeiro programa, uma vez que basta, neste caso, considerar o ângulo de inclinação sendo $90^{\circ}$.

Os algoritmos desenvolvidos e implementados em linguagem Pascal tratam-se de uma ferramenta que poderá vir a ser utilizada na resolução dos problemas reais da indústria metalúrgica.

\section{REFERÊNCIAS}

ARAUJO, E. C. Curso Técnico de Caldeiraria. São Paulo: Hemus, 1976.

BESANT, C.B. CAD/CAM Projeto e fabricação com auxílio do computador. Trad. de Ricardo Reinprecht. $3^{\text {a }}$ edição. Rio de Janeiro:Campus, 1988. 249p.

BASSANEZI, R. C. , "Ensino aprendizagem com modelagem matemática: uma nova estratégia”, São Paulo: Contexto, 2002.

BIEMBENGUT, Maria Salete. Modelagem Matemática \& implicações no ensino e aprendizagem da matemática. 2 ed. Blumenau: Furb, 1999.

CALDSoft: software comercial para planificações em caldeiraria industrial. Disponível em:

<http://www.caldsoft.com.br>. Acesso em: maio 2013.

CASTRO, M. M. .O. Método Analítico para Planificação de Interseções entre Superfícies Cilíndricas e Cônicas. Niterói: Universidade Federal Fluminense EDUFF, 1991. v. 1 e 2, 628 p.

COOKSON, W. New Methods for Sheet Metal Work. Londres: The Technical Press, 1964. 227 p.

GOLDBARG, M.C.; LUNA, H.P. Otimização combinatória e Programação Linear. $2^{\mathrm{a}}$ Edição, Rio de Janeiro: Elsevier, 2005, 520 p. 
MAZIERO, N. L.; Oro, N. T.; Kripka, R. M. L.. Planificação de superfícies: seccionamento de peças. XXIV Iberian Latin-American Congress on Computational Methods in Engineering, Ouro Preto/MG Brazil, 2003.

MAZIERO, Nilson L.; Oro, Neuza T.; Kripka, M. L. Planificação de Superfícies: Aplicação de um modelo em um sistema CAD. Ciência \& Engenharia, Uberlândia, v. 12, n. 1, p. 61-66, 2003.

ORO, N.T., KRIPKA, R.M.L., MAZIERO, N. L. Modelagem e resolução do Processo de Planificação de sólidos. Tendências em matemática Aplicada e Computacional (TEMA), Rio de Janeiro ,v.1, p. 201-214, dezembro2000.

PERTENCE, A. E.; de Mello Júnior, L. M. L. Desenvolvimento de um programa didático para cálculo automatizado do processo de planificação de superfícies. Revista de Ensino de Engenharia, v.19, n.1, p.11-17, 2000. 